\title{
Independent Component Thermography for Subsurface Defect Detection
}

\author{
by Jin-Yi Wu*, Stefano Sfarra**, Hsiu-Li Wen*, Yuan Yao*† \\ * Department of Chemical Engineering, National Tsing Hua University, Hsinchu, 30013, Taiwan, ROC, \\ ** Department of Industrial and Information Engineering and Economics, University of L'Aquila, Piazzale E. \\ Pontieri no. 1, Monteluco di Roio - L'Aquila (AQ), I-67100, Italy \\ † Corresponding author: yyao@mx.nthu.edu.tw
}

\begin{abstract}
In this work, the concept of blind source separation (BSS) is introduced into the field of thermographic data processing to facilitate subsurface defect detection. In detail, the thermal images generated by infrared thermography are viewed as a set of mixed signals of multiple independent and non-Gaussian sources, which can be decomposed by using the proposed independent component thermography (ICT) technique. The decomposition results highlight the locations and shapes of the defective regions by eliminating the effects of backgrounds. The feasibility of ICT is illustrated with its application to the analysis of the conservation status of a panel painting
\end{abstract}

\section{Introduction}

Infrared thermography (IRT) is a popular tool for non-destructive testing (NDT), which is cost-effective, easy-tosetup, and able to rapidly inspected large areas. The principle of IRT is based on the detection of thermal radiation emitted from the surface of the investigated objects. Generally, IRT can be divided into active IRT (AIRT) and passive IRT (PIRT). AIRT requires an external heat source to stimulate the tested materials, while in PIRT, the features to identify are naturally at a different temperature than the background radiation. For the applications of material defect detection, AIRT is a more suitable technique.

Despite of many advantages, IRT may generate thermal images difficult to be segmented. The main reason is the existence of noise and inhomogeneous background. In addition, the amount of thermal images produced by IRT is usually very large. Therefore, it is difficult to visually inspect each of them. To solve these problems, a number of thermographic data processing methods have been proposed, such as principal component thermography (PCT) [1], pulsed phase thermography (PPT) [2], thermographic signal reconstruction (TSR) [3], higher-order statistics (HOS) [4], pixelwise algorithm for time-derivative of temperature (PATDT) [5, 6], and so on. However, the concept of blind source separation (BSS) has seldom been adopted. In IRT, the generated thermal images can be regarded as a set of mixed signals of multiple sources. If they can be decoupled in a reasonable way, the defective regions may be easily identified. The potential of BSS for thermographic data analysis motivates this research.

\section{Independent component thermography (ICT)}

The method proposed in this paper is named independent component thermography (ICT), which is based on one of the most popular BBS algorithms, independent component analysis (ICA) $[7,8]$. In the first step of ICT, each thermal image is vectorized. As a result, the entire three-dimensional (3-D) thermographic data set is transformed to a twodimensional (2-D) matrix $\mathbf{X}$ as illustrated in Fig.1, where $I$ is the total number of frames, and $J \times K$ is the dimensions of each thermal image. Without loss of generality, suppose that $\mathbf{X}$ has been column-wise centered by subtracting the mean of each pixel. Then, ICA is conducted to decompose $\mathbf{X}$ into a number of independent components (ICs), i.e. source signals. The hidden assumptions behind this algorithm include that 1) the source signals are independent of each other, and 2) the values in each source signal are non-Gaussian distributed. In the mathematical expression, each column of $\mathbf{X}$, denoted as $\mathbf{x}(k)=\left[x_{1}(k), \ldots, x_{I}(k)\right]^{T}$, can be viewed as a linear combination of multiple ICs, i.e.

$$
\mathbf{x}(k)=\mathbf{A s}(k)+\mathbf{e}(k),
$$

where $\mathbf{A}$ is the mixing matrix with dimensions of $I \times M, M$ is the number of ICs, $\mathbf{s}(k)=\left[s_{1}(k), \ldots, s_{M}(k)\right]^{T}$ is vector of ICs, $\mathbf{e}(k)$ is residual vector, and $k=1, \ldots, J K$. When $M$ is chosen to be equal to $I, \mathbf{e}(k)$ becomes a zero vector. Then, the decomposition can be expressed as

$$
\mathbf{X}=\mathbf{A S}
$$

where $\mathbf{S}$ is the IC matrix composed of $\mathbf{s}(k), k=1, \ldots, J K$ and can be estimated using Eq. (3).

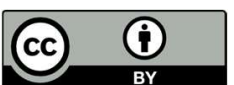



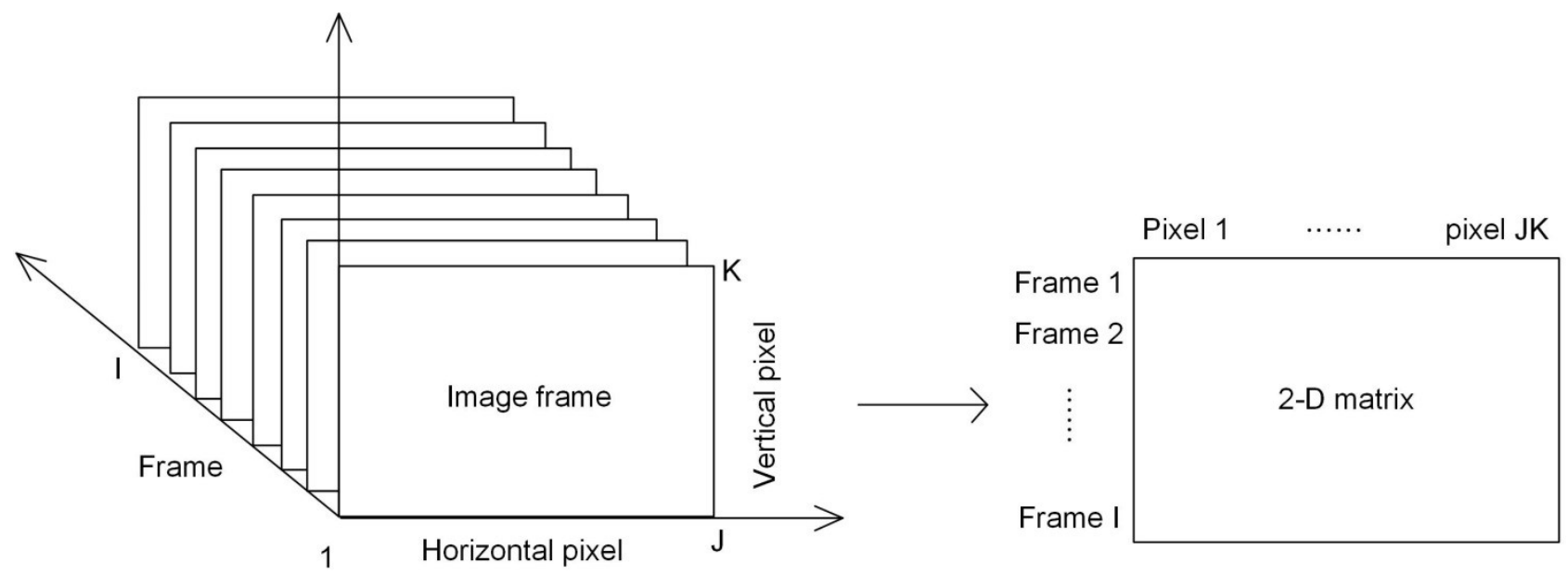

Fig .1. Thermographic data unfolding

$$
\hat{\mathbf{S}}=\mathbf{W X},
$$

where $\mathbf{W}$ is the de-mixing matrix. In order to calculate $\mathbf{W}$, the ICs are constrained to have unit variance, i.e.

$$
E\left[\mathbf{s}(k) \mathbf{s}^{T}(k)\right]=\mathbf{I} .
$$

In addition, whitening is a requested pre-processing step, aiming to remove the cross-correlation between the signals. Such a transformation can be written as

$$
\mathbf{z}(k)=\mathbf{Q x}(k),
$$

where $\mathbf{Q}$ is the whitening matrix that can be calculated by $\mathbf{E q}$. (6):

$$
\mathbf{Q}=\boldsymbol{\Lambda}^{-1 / 2} \mathbf{U}^{T} .
$$

Here, $\boldsymbol{\Lambda}$ is a diagonal matrix containing all the eigenvalues of the covariance matrix of the original signals, and $\mathbf{U}$ is the corresponding eigenvector matrix. Suppose that the following condition is satisfied:

$$
E\left(\mathbf{z}(k) \mathbf{z}^{T}(k)\right)=\mathbf{I} .
$$

Eq. (8) can be derived.

$$
\mathbf{z}(k)=\mathbf{Q} \mathbf{x}(k)=\mathbf{Q A s}(k)=\mathbf{B s}(k),
$$

where $\mathbf{B}=\mathbf{Q A}$ is an orthogonal matrix. Thus, the problem is transformed to finding the matrix $\mathbf{B}$. Consequently, the demixing matrix can be calculated as

$$
\mathbf{W}=\mathbf{B}^{T} \mathbf{Q} .
$$

The problem can be solved by maximizing the non-Gaussianity which is quantified by the kurtosis, the measure of the "tailedness" of the probability distribution. For more details of the algorithm, please refer to the literature [7, 8]. In the third step, each IC can be reshaped to the dimensions of the original thermal images, facilitating the identification of the defective regions. Usually, the ICs corresponding to the high kurtosis values have large chances to reveal the defects, because the existence of the defects changes the material thermal conductivity and increases the non-Gaussianity of the corresponding pixel values in the thermal images.

\section{Case study}

In the case study, the proposed ICT method is utilized to analyze the conservation status of a panel painting named "Madonna" and made of poplar wood. The dimensions of the painting are $15 \times 21 \times 2 \mathrm{~cm}$. Four fabricated defects ( $A, B, C$ and $D$ ) with various shapes and sizes were produced by inserting Mylar sheets at different depths inside the panel, as shown in Fig. 2. The specifications of the defects are shown in Table 1. 


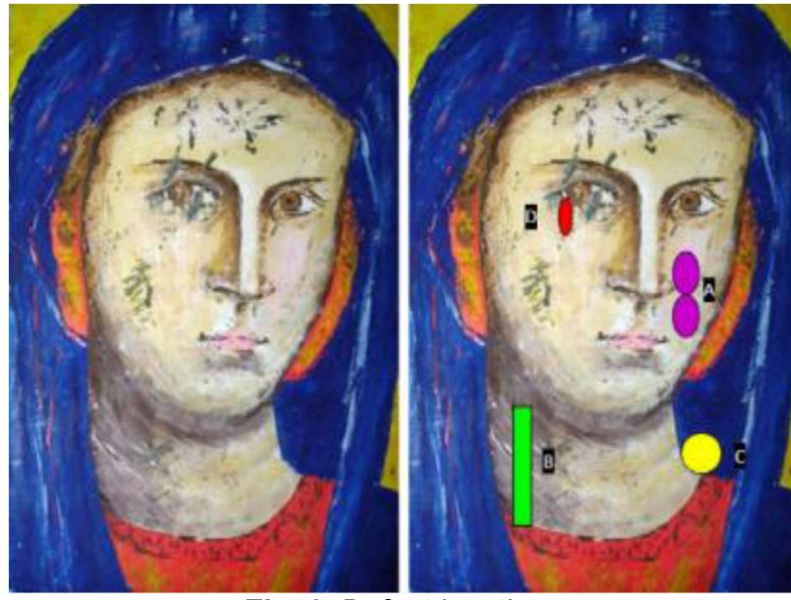

Fig. 2. Defect locations

Table 1. Specification of defects [9]

\begin{tabular}{|c|c|c|}
\hline Defect & Dimensions $(\mathrm{mm})$ & Depth range $(\mathrm{mm})$ \\
\hline A & Diameter $=5$ & $0.3-0.8$ \\
\hline B & $28 \times 4$ & $0.8-0.9$ \\
\hline C & Diameter $=8$ & $>1.4$ \\
\hline D & $14 \times 3$ & $>2.0$ \\
\hline
\end{tabular}

In the experiment, the panel was tested by using active IRT, where two $250 \mathrm{~W}$ lamps (Siccatherm E27, OSRAM) were adopted as the energy sources. The distance between the painting and the center of each lamp was $48 \mathrm{~cm}$, while the two lamps were separated by a distance of $50 \mathrm{~cm}$. An infrared camera (ThermaCAM S65HS, FLIR) with a resolution of $320 \times 240$ pixels was used to capture the thermal images, which was setup in front of the painting at a distance of 63 $\mathrm{cm}$. The sampling rate was $1 \mathrm{frame} / \mathrm{sec}$. Several raw thermal images collected during the experiment are shown in Fig. 3 , from which it is difficult to identitify the defects by visual inspection. Furthermore, it is labor extensive to examine the large amount of thermal images one by one. Therefore, thermographic data processing is a necessity. In this study, the analyses were applied to the images collected during the heating phase, because the changes of the material surface temperature during this phase is significant.

For the sake of comparison, PCT [1] and TSR [3] were implemented. The results are shown in Fig. 4 - Fig. 6. PCT achieves data compression and highlights the defects in the third, fourth, and sixth empirical orthogonal functions (EOFs) which are denoted as PC3, PC4, and PC6 in Fig. 4. However, PCT does not separate the defects and the pigments, making it difficult to achieve defect identification in a direct way. TSR performs much worse than PCT. Fig. 5 shows the TSR results, while Fig. 6 shows the TSR signals with first time derivatives. Comparing to the orignal thermal images, TSR does not significantly improve the contrast between the defective and intact regions. In this case study, the order of the polynomial was selected to be $n=4$.

Then, ICT was conducted. Fig. 7 plots the values of the kurtosis statistic corresponding to different ICs, where a larger kurtosis value indicates more significant non-Gaussianity of the corresponding IC. Several ICs with different degrees of non-Gaussianity were reconstructed into images, as shown in Fig. 8. It is clear that the IC with the highest kurtosis, i.e. IC5, indicates the positions of Defects A, B and C clearly, while the the effects of oil pigments and noises are contained in other IC images. In this way, the information from different sources are separated, facillitating the identification of the sursurface defects. It is noted that the deepest defect, i.e. Defect $D$, is not clearly identified by any of the methods used in this study. A possible reason is that the information of this defect is not contained in the original thermal images because of its depth.

In Table 2, the index of signal-to-noise ratio (SNR) [10, 11], which is defined as

$$
S N R=\frac{\left|M_{D}-M_{I}\right|}{\sigma_{I}},
$$

is adopted to evaluate the performance of these methods in a more objective manner. Here, $M_{D}$ and $M_{I}$ are the average values of the pixels located in the defective region and intact region, respectively, and $\sigma_{I}$ represents the standard deviation of the pixel values in the intact region. This dimensionless index quantifies the signal contrast between the defective and non-defective regions. Usually, large SNR values are desired. In Table 2, the highest SNR values achieved by each method on different defects are listed. It is clear that the proposed ICT method has the best performance in average. For the identification results based on the original thermal images or TSR, the numbers in the brackets indicate the sampling time points when the frame was taken. For the results of PCT and ICT, the characters in the brackets denotes the index values of EOFs or ICs based on which the best SNR values were obtained. It is noted that none of the methods can detect Defect $D$ effectively. All the SNR values corresponding to this defect are smaller than 1 , indicating that it is impossible to 
differentiate the defect information from noise.
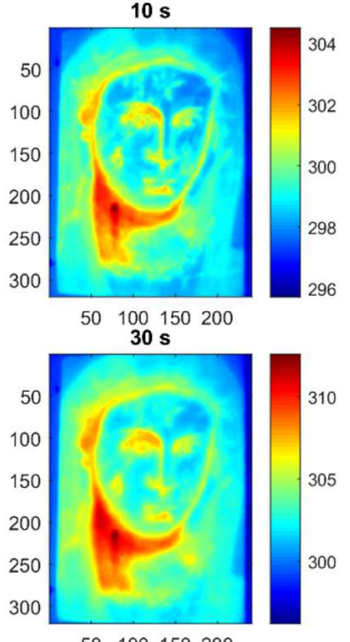

100150200
$80 \mathrm{~s}$

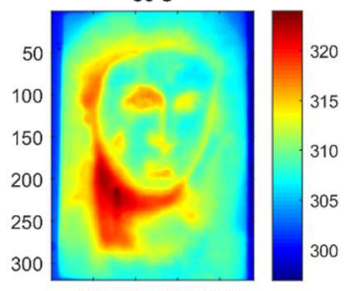

50100150200

Fig. 3. Original thermal images

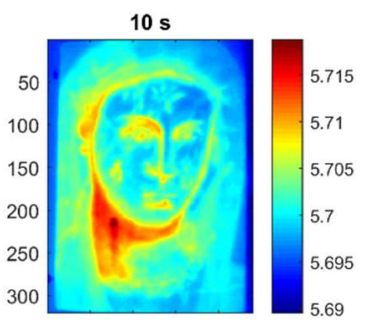

50100150200
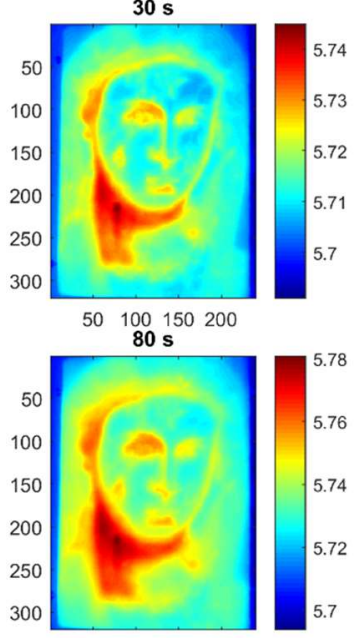

50100150200

Fig. 5. TSR results
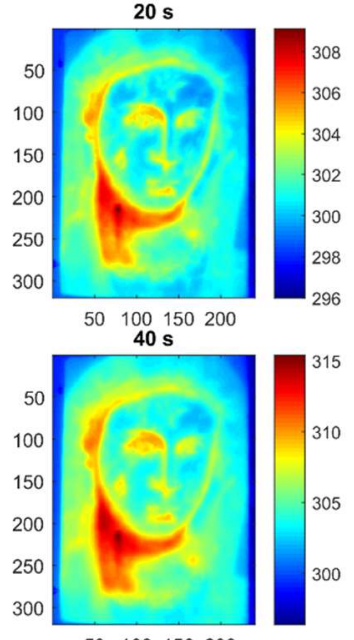

$50 \underset{900}{100} 150200$

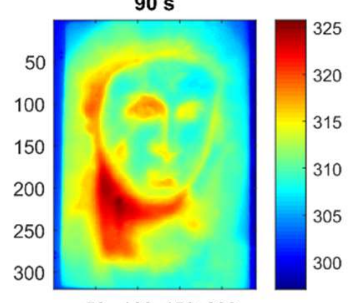

50100150200

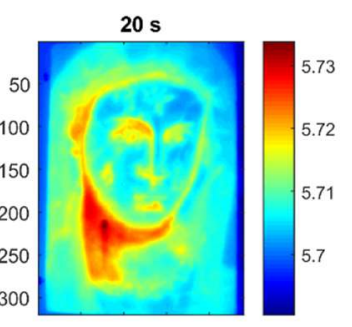

50100150200

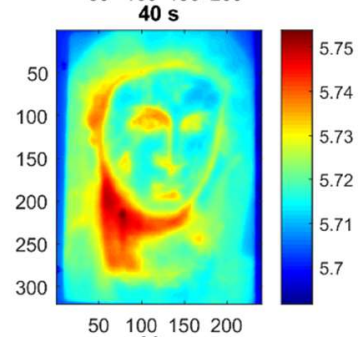

$50 \quad 100150200$

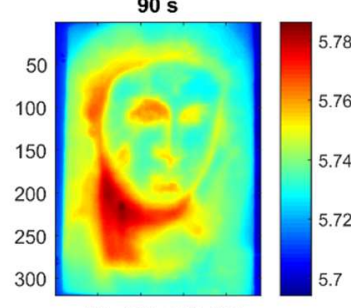

50100150200

5.7
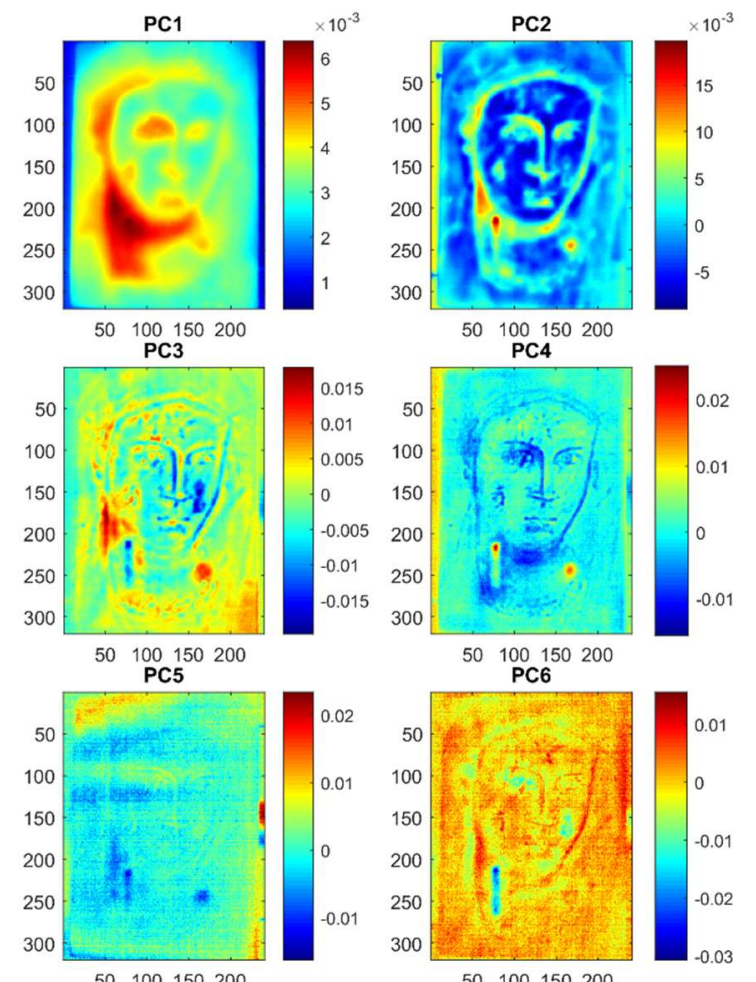

Fig. 4. PCT results
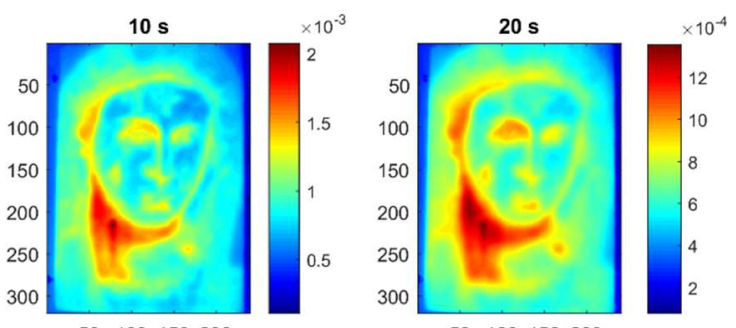

100150200

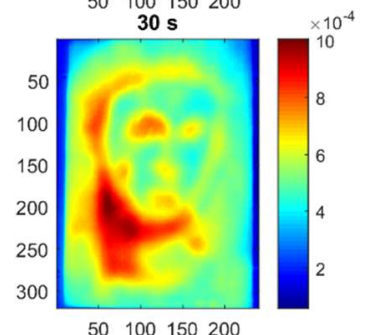

50100150200
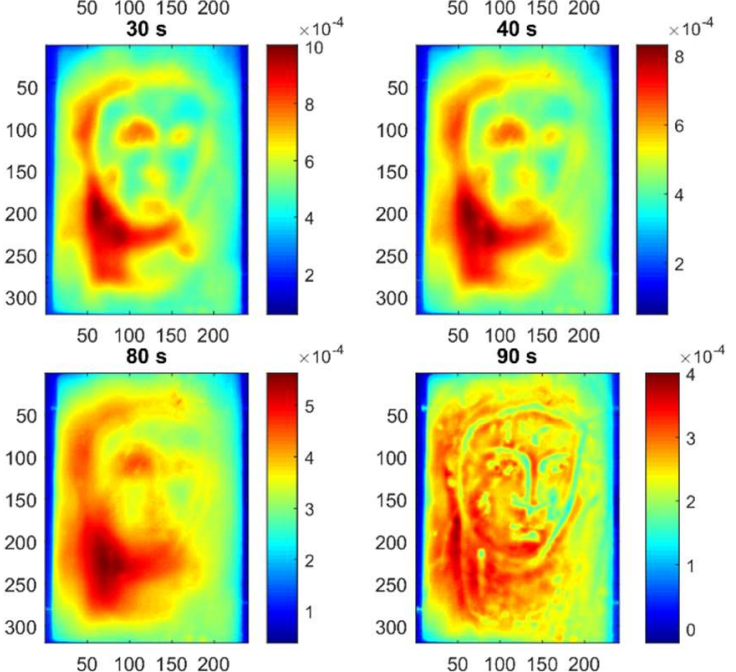

Fig. 6. TSR (with first order derivative) results 


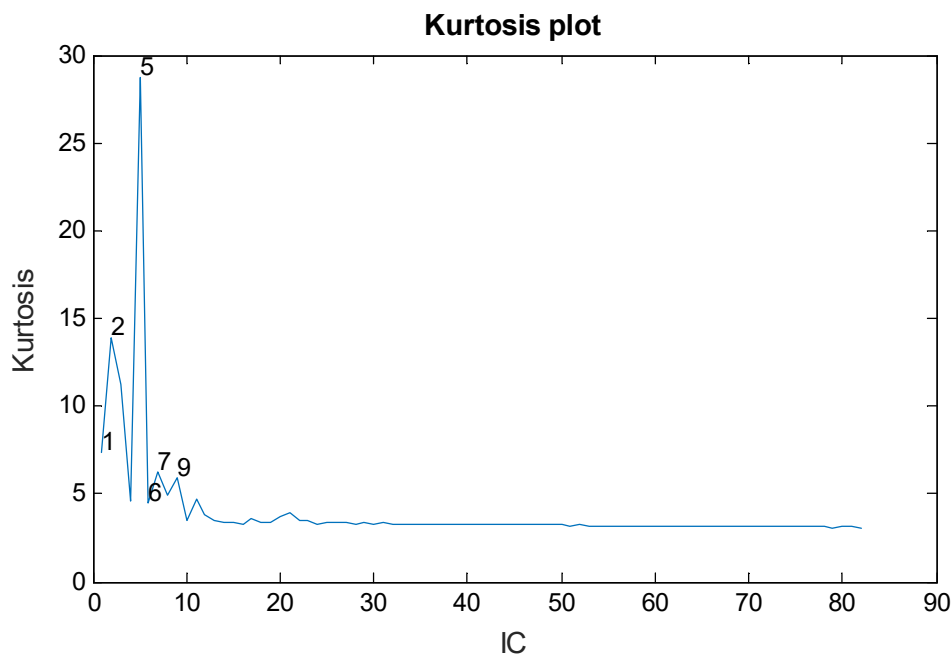

Fig. 7. Kurtosis plot
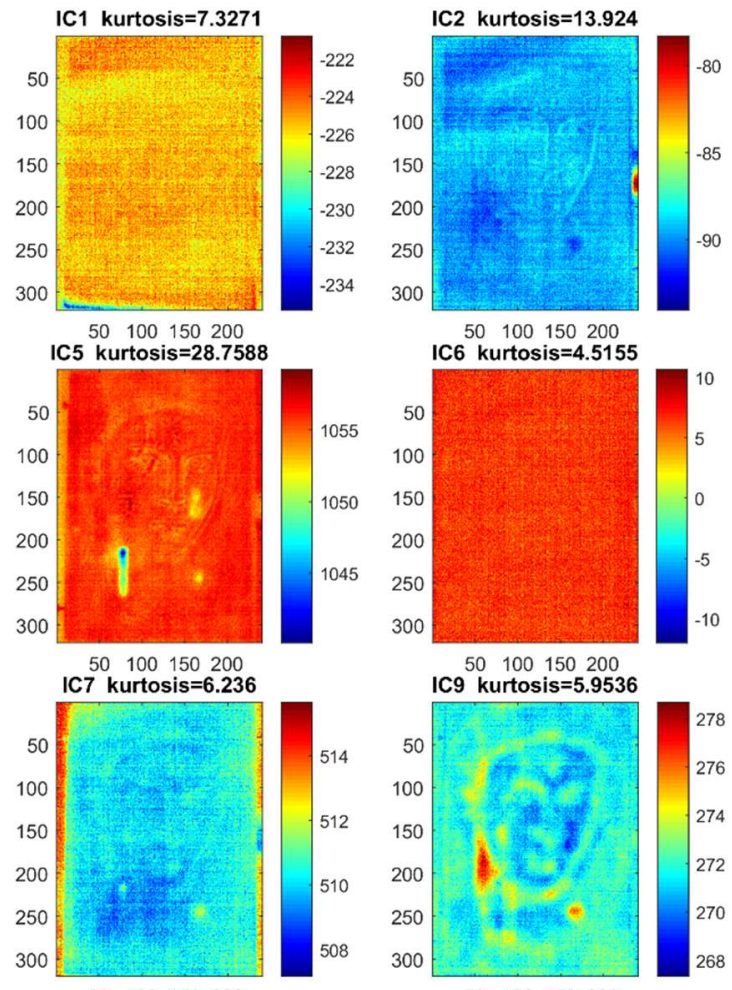

Fig. 8. ICT results

Table 2. Comparison of SNR values

\begin{tabular}{|c|c|c|c|c|}
\hline SNR & Defect A & Defect B & Defect C & Defect D \\
\hline Original thermal images & $0.237(1.8 \mathrm{~s})$ & $2.810(0.333 \mathrm{~s})$ & $0.973(0.967 \mathrm{~s})$ & $0.843(0.2 \mathrm{~s})$ \\
\hline PCT & $3.282(P C 3)$ & $4.081(\mathrm{PC} 6)$ & $2.918(\mathrm{PC} 3)$ & $0.967(\mathrm{PC} 5)$ \\
\hline TSR $(\mathrm{n}=4)$ & $0.231(1.8 \mathrm{~s})$ & $2.782(0.367)$ & $2.315(0.033 \mathrm{~s})$ & $0.850(0.067 \mathrm{~s})$ \\
\hline TSR(1-derivative) & $0.498(3 \mathrm{~s})$ & $3.052(0.033 \mathrm{~s})$ & $1.221(0.033 \mathrm{~s})$ & $0.832(0.733 \mathrm{~s})$ \\
\hline ICT & $1.975(\mathrm{IC5})$ & $6.959(\mathrm{IC} 5)$ & $3.005(\mathrm{IC9})$ & $0.956($ IC2) \\
\hline
\end{tabular}




\section{Conclusions}

In this research, the ICT method is proposed for thermographic data processing. By decomposing the sequence of thermal images generated by IRT into a number of independent and non-Gaussian source signals, different types of information can be separated. Such a feature of ICT significantly facilitates the defect identification based on the visualized decomposition results, making it outperforms the conventional methods, such as PCT and TSR, in the case study on a panel painting. It is noted that there is no dominant approach in thermographic data processing. The choice of methods is often case dependent. In the future research, a comprehensive comparison of different thermographic data analysis methods, from both the mathematical and engineering viewpoints, is desired.

\section{Acknowledgments} 013-MY2.

This work was supported by Ministry of Science and Technology, ROC under Grant No. MOST 105-2628-E-007-

\section{REFERENCES}

[1] Rajic, N., Principal component thermography for flaw contrast enhancement and flaw depth characterisation in composite structures. Composite Structures, 2002. 58(4): p. 521-528.

[2] Maldague, X. and S. Marinetti, Pulse phase infrared thermography. Journal of applied physics, 1996. 79(5): p. 2694-2698.

[3] Vavilov, V., et al., Thermal nondestructive testing of carbon epoxy composites: detailed analysis and data processing. NDT \& E International, 1993. 26(2): p. 85-95.

[4] Madruga, F.J., et al., Infrared thermography processing based on higher-order statistics. NDT \& E International, 2010. 43(8): p. 661-666.

[5] Rodríguez-Martín, M., et al., Cooling analysis of welded materials for crack detection using infrared thermography. Infrared Physics \& Technology, 2014. 67: p. 547-554.

[6] Rodríguez-Martín, M., et al., Crack-depth prediction in steel based on cooling rate. Advances in Materials Science and Engineering, 2016. 2016.

[7] Hyvärinen, A. and E. Oja, Independent component analysis: algorithms and applications. Neural networks, 2000. 13(4-5): p. 411-430.

[8] Hyvarinen, A., Fast and robust fixed-point algorithms for independent component analysis. IEEE transactions on Neural Networks, 1999. 10(3): p. 626-634.

[9] Grinzato, E., et al., Non-destructive testing of wooden painting by IR thermography. ECNDT, 8th, Barcelona, 2002.

[10] Ibarra-Castanedo, C., et al., Comparative study of active thermography techniques for the nondestructive evaluation of honeycomb structures. Research in Nondestructive Evaluation, 2009. 20(1): p. 1-31.

[11] Zheng, K., Y.-S. Chang, and Y. Yao, Defect detection in CFRP structures using pulsed thermographic data enhanced by penalized least squares methods. Composites Part B: Engineering, 2015. 79: p. 351-358. 